\title{
Lung morphology and surfactant function in cardiogenic pulmonary edema: a narrative review
}

\author{
Kenneth Nugent, Logan Dobbe, Rubayat Rahman, Mohamed Elmassry, Pablo Paz \\ Department of Internal Medicine, Texas Tech University Health Sciences Center, Lubbock, TX, USA \\ Contributions: (I) Conception and design: All authors; (II) Administrative support: None; (III) Provision of study materials or patients: None; (IV) \\ Collection and assembly of data: All authors; (V) Data analysis and interpretation: All authors; (VI) Manuscript writing: All authors; (VII) Final \\ approval of manuscript: All authors. \\ Correspondence to: Kenneth Nugent, MD. $36044^{\text {th }}$ Street, Lubbock, TX 79430, USA. Email: kenneth.nugent@ttuhsc.edu.
}

\begin{abstract}
The conventional analysis of acute cardiogenic pulmonary edema involves the development of high pulmonary capillary pressures resulting in hydrostatic gradients for fluid flux out of capillaries into the interstitial space and alveolar spaces. However, some patients respond poorly to diuretic management. The PubMed database was searched to identify experimental studies on pulmonary edema in animals, experimental studies on surfactant function, including patients with pulmonary edema, and clinical studies reporting barrier dysfunction and/or injury in patients with acute pulmonary edema. Studies with animal models demonstrate that high capillary pressures can cause barrier disruption in alveolar capillary units which increases permeability and the transfer of fluid and protein into lung parenchyma. Fluid in alveolar spaces alters surfactant function which increases fluid flux out of capillaries into the lung parenchyma secondary to larger transcapillary hydrostatic gradients. Patients with acute cardiogenic pulmonary edema have increased levels of surfactant protein B in their plasma which reflect barrier disruption and increased levels of tumor necrosis factor alpha which reflect acute tissue injury. Increased surfactant protein B plasma levels are associated with abnormal gas exchange in patients with chronic heart failure. Patients with exercise-induced left ventricular dysfunction have increased levels of surfactant protein B after short periods of exercise. Pathology studies in patients with chronic heart failure have found increased connective tissue in alveolar capillary units and increased numbers of type II alveolar cells, and these changes represent an adaptive response in these patients. Clinicians need to consider the possibility of barrier dysfunction and disruption in patients with both acute and chronic pulmonary edema and understand that diuresis may have a limited effect on symptoms in some patients.
\end{abstract}

Keywords: Pulmonary edema; surfactant; capillary pressure; alveolar capillary permeability; morphology

Submitted Apr 06, 2019. Accepted for publication Aug 28, 2019.

doi: $10.21037 /$ jtd.2019.09.02

View this article at: http://dx.doi.org/10.21037/jtd.2019.09.02

\section{Introduction}

The conventional analysis of acute cardiogenic pulmonary edema involves the development of high pulmonary capillary pressures in the lung resulting in a hydrostatic gradient for fluid flux out of capillaries into the interstitial space and alveolar spaces (1). However, this analysis does not consider disruptions in the blood gas barrier which can develop from high pressures in pulmonary capillaries and the effect of pulmonary edema on surfactant function (2). Studies in patients and animal models, discussed below, demonstrate that high capillary pressures cause lung injury and possibly lung inflammation, and these events need consideration when managing patients with cardiogenic pulmonary edema. In this review we discuss studies relevant to lung anatomy, experimental hydrostatic pulmonary edema, experimental studies with surfactant, clinical studies on surfactant, surfactant release during episodes of 
pulmonary edema, and human pathology in patients with chronic pulmonary edema. This review will help clinicians understand the formation of pulmonary edema and help explain the clinical course in some patients who respond poorly to diuretics.

\section{Lung anatomy}

The adult lung has approximately 480 million alveoli with an average size of $4.2 \times 10^{6} \mu^{3}$ (3). Alveoli have a polyhedral shape which changes in size and configuration during inflation and deflation of the lung. The average diameter is in the range of $160 \mu \mathrm{m}$. The total alveolar surface area is approximately $130 \mathrm{~m}^{2}$; the total capillary surface area is approximately $115 \mathrm{~m}^{2}$ with a capillary volume of $200 \mathrm{~mL}$. Alveolar type I cells cover $95 \%$ of the internal alveolar surface, and alveolar type II cells cover the remaining $5 \%$. Alveolar type II cells secrete surfactant which lowers the surface tension in the alveoli. Surface tension increases during inflation and decreases during deflation due to simple mechanical stretching of elastic elements. Hysteresis is a difference in the tension-length (or pressure-volume) curve depending on the direction of motion. Tension or pressures are higher for any degree of stretch or volume during inflation than during deflation. Surfactant decreases surface tension at all volumes and in both directions, but not by the same magnitude in each direction. The average alveolar fluid depth is in the range of $0.14-0.2 \mu \mathrm{m}$. The alveolar epithelium is in close proximity to alveolar capillaries, and approximately $70 \%$ of alveolar surfaces are associated with capillary networks (4). The width of the blood gas barrier varies from $2-3 \mu \mathrm{m}$ in "thick" regions to $0.2-0.3 \mu \mathrm{m}$ in "thin" regions. Thin regions have little or no interstitial space, and the basement membranes are fused.

This large surface area with small distances between alveoli and capillaries facilitates gas exchange through diffusion. However, this large network is at risk for mechanical injury secondary to both increased alveolar and transpulmonary pressures and increased capillary pressures. Surfactant has an essential role in maintaining alveolar stability, especially at low lung volumes. Without the hysteresis effect of surfactant on the pressure-volume curve for the lung, deflation of the alveoli would yield more energy than the work required to inflate other alveoli, and all alveoli would spontaneously empty into a single alveolus. Furthermore, in the absence surfactant, the pressure required to inflate alveoli is too high. Consequently, surfactant deficiency or surfactant dysfunction can cause reduced lung volumes (atelectasis) and abnormal gas exchange. The usual analysis of cardiogenic pulmonary edema considers this a disorder associated with increased interstitial and alveolar fluid based on Starling mechanisms. However, increased capillary pressure can cause stress capillary failure, and alveolar fluid can cause surfactant dysfunction and increase surface tensions. These events need consideration when managing patients with cardiogenic pulmonary edema. In this review we will discuss experimental and clinical studies on lung morphology and surfactant relevant to cardiogenic pulmonary edema.

\section{Lung morphology in experimental hydrostatic pulmonary edema}

Bachofen and coworkers studied lung morphology in rabbits with experimental hydrostatic pulmonary edema created by perfusing excised lungs with $6 \%$ albumin solutions at either high filtration pressures $(29 \mathrm{mmHg})$ or low filtration pressures (14 mmHg) (5). Lungs perfused at higher pressures rapidly developed edema and gained weight. Studies with light microscopy and transmission and scanning electron microscopy demonstrated that the distribution of alveolar edema and interstitial edema was not homogeneous, even at the same lung height. Albumin concentrations also varied in the alveoli and interstitial spaces. There was a gradient of pulmonary edema which increased from the apical to basal segments of the lung in the upright lung but not in the inverted lung. In some but not all regions there was an osmiophilic layer (surfactant) on the edema fluid.

These investigators also made a detailed analysis of barrier lesions in rabbit lungs with experimental hydrostatic pulmonary edema and found numerous breaks in the epithelial cell layer (6). In high pressure edema, there were disruptions in the thin and thick side of the blood gas barrier; in moderate pressure edema, epithelial cells developed blebs which varied significantly in size. Endothelial lesions were uncommon in both high and moderate pressure experiments. Barrier lesions were seen exclusively in regions with alveolar edema. This result would suggest that barrier leaks have a role in hydrostatic edema formation. These barrier lesions developed within 30 min in these experimental lung preparations and can close rapidly (see text below).

West and coinvestigators have studied pulmonary capillary stress failure in animals and humans (4). They noted that the blood gases barrier has a remarkable area but minimal thickness and that capillaries must withstand 
high stresses during severe exercises. Stresses on the blood gas barrier include increased circumferential tension caused by high capillary transmural pressures and longitudinal tension in alveolar walls associated with lung inflation; the surface tension from alveolar lining fluid may have some effect on capillaries depending the lung volume, lung volume history, and capillary pressures. The strength of the blood gas barrier comes from the extracellular matrix, predominantly from type IV collagen. Scanning electron microscopy studies have demonstrated that disruptions occur in both the endothelial cells and epithelial cells and do not necessarily occur at intracellular junctions (7). These studies used a rabbit model with an open chest and cannulas in the pulmonary artery and left atrium. The transmural capillary pressures ranged from 12.5 to $72.5 \mathrm{~cm}$ of $\mathrm{H}_{2} \mathrm{O}$. The number of breaks in the capillary endothelium and the alveolar epithelium increased significantly at higher pressures. However, in many areas with endothelial and epithelial disruptions, the basement membranes remained intact. At higher pressures the width of the blood gas barrier increased secondary to interstitial edema.

A second series of experiments with this model demonstrated that high lung volumes increased stress failure in pulmonary capillaries (8). In these experiments, the lungs of anesthetized rabbits were inflated to a transpulmonary pressure of $20 \mathrm{~cm}$ of $\mathrm{H}_{2} \mathrm{O}$ and then perfused with blood at either 32.5 or $52.5 \mathrm{~cm}$ of $\mathrm{H}_{2} \mathrm{O}$ pressure transmural capillary pressure. These results were compared to studies with the transpulmonary pressures held at $5 \mathrm{~cm}$ of $\mathrm{H}_{2} \mathrm{O}$. There was a significant increase in the number of endothelial breaks and epithelial breaks in animals with high transpulmonary pressures. A third series of experiments demonstrated that there was a significant decrease in the number of endothelial and epithelial breaks when the pressure was lowered from 52.5 to $12.5 \mathrm{~cm}$ of $\mathrm{H}_{2} \mathrm{O}(9)$. These studies were repeated using lung preparations filled with normal saline to alter the surface tension in the alveoli. In comparison with air-filled lungs, there were a large number of breaks in the inner boundary of the epithelium. There was no change in the number of disruptions in the endothelium, and there were fewer disruptions in the outer boundaries of epithelial cells. In summary, these studies demonstrate that there is a significant disruption in the blood gas barrier in lung preparations with high transcapillary pressures, the number of disruptions increases at higher lung volumes, these ruptures reverse quickly, and alterations in surfactant activity on the alveolar surface change the site of disruptions.
West et al. studied lung injury in race horses and elite athletes at extreme work loads $(10,11)$. Hopkins et al. studied a lung injury in elite athletes at extreme workloads (11). These athletes had increased numbers of erythrocytes and increased amounts of total protein and leukotriene B in their bronchoalveolar lavage fluid collected one hour after a seven-minute cycling race simulation. There was no increase in surfactant protein A, tumor necrosis factor alpha activity, lipopolysaccharides, or interleukin-8.

In summary, high capillary pressures cause disruptions in the alveolar capillary unit which increase the transfer of fluid into the interstitial spaces and alveolar spaces. The models used by Weibel et al. and West et al. demonstrate that the fluid collection is not homogeneous in the lung and barrier disruptions can recover quickly. The accumulation of fluid in alveolar spaces can cause surfactant dysfunction and increase surface tension. Stress failure also occurs in clinical conditions, including high altitude pulmonary edema, neurogenic pulmonary edema, severe left ventricular failure, and mitral stenosis.

\section{Experimental studies on surfactant}

Said measured pulmonary surface activity in lung extracts from dogs with experimental pulmonary edema induced by the rapid infusion of dextran (12). The tension of the surface film was measured continuously and recorded as a function of surface area. The minimum surface tension and the maximum surface tension increased in edematous regions of the lung. This was explained by the loss of the surfactant into the edema foam created in these dogs' lungs. These investigators noted that patchy atelectasis developed in the edematous lungs which they attributed to the loss of surfactant activity and that pressure-volume relationships were abnormal in the excised lungs with pulmonary edema.

Albert studied the effect of changes in surface tension on the formation of pulmonary edema in anesthetized dog lungs (13). These investigators used an open chest dog model to determine the isogravimetric pressure that resulted in a net fluid flux of 0 across the capillary into the lung, determined by constant monitoring of lung weight. The alveolar surface tension was increased by cooling the lung; this increased the alveolar pressure needed to maintain control lung volumes and was reversible when the lung was rewarmed. With rewarming, the surface tension decreased, and the pressure needed to maintain constant lung weight increased and was closer to alveolar pressure. Consequently, this model demonstrates that 
increased surface tension favors fluid leakage because the microvascular pressure gradient from the lung capillary to the interstitium increases. A decrease in interstitial perimicrovascular pressure presumably reflects the changes in surface tension, and increases in surface tension cause a more negative interstitial pressure which increases fluid flux out of the capillaries.

Bredenberg et al. studied the effect of increased surface tension on the formation of pulmonary edema in a dog model. These animals were exposed to a detergent (dioctyl sodium sulfosuccinate) by nebulization, and extravascular water volumes in the lung increased (14). This was associated with a loss of surfactant activity and increased alveolar surface tension measured in lung tissue. The animals also had decreased static compliance and atelectasis. There was no significant change in either colloid oncotic pressure or pulmonary microvascular pressure to explain these results. These investigators suggested pulmonary edema was caused by increased alveolar surface tension. In another series of experiments, these investigators cannulated the tracheobronchial lymphatic system and measured the protein concentration in lymph flow after increasing left atrial pressure (15). Animals exposed to the aerosol detergent had a reduction in the protein concentration in the lymph fluid. In addition, measurement of the protein concentration in airway fluid suggested that there was no increase in microvascular permeability during these experiments. These investigators completed a third series of experiments in which animals were exposed to the detergent aerosol and then ventilated with either $5 \mathrm{~cm}$ of water positive end-expiratory pressure (PEEP) or $10 \mathrm{~cm}$ of water PEEP (16). Animals managed with higher levels of PEEP had increased extravascular lung water. The authors suggested that the increased PEEP level changed pressure gradients across extra-alveolar vessels located in alveolar corners and this increased the hydrostatic capillary pressure gradient from the capillaries to the interstitium.

The studies reported by Said and Albert suggest that pulmonary edema changes surfactant activity in alveolar spaces and increases surface tension. This leads to atelectasis and hypoxemia. In addition, increased surface tension can change pressure gradients in alveolar capillary units and increase fluid flux out of capillaries into the interstitial space and the alveolar space which increases the formation of more edema. The studies reported in dogs indicate that an increase in alveolar surface tension increases edema formation and that higher PERP levels increase the rate of formation. The studies indicate that pulmonary edema can have an important adverse effects on surfactant function, and these adverse effects are potentially relevant to patients with cardiogenic pulmonary edema.

Smallwood et al. demonstrated that short-term exposure of surfactant to $100 \%$ oxygen had a significant effect on its surface tension lowering ability and reduced the size surfactant particles in the aqueous phase below the surfactant level (17). This occurs because surfactant molecules are lost into aqueous subphase during the repeated cycling (i.e., surface area changes) in these experiments. This study suggests that the exposure of surfactant to high concentrations of oxygen might reduce its function and potentially contributes to the lack of benefit with exogenous surfactant in studies with adults with acute respiratory distress syndrome. More important, it might suggest that high concentrations of oxygen in patients have adverse effects on surfactant which, in turn, increase edema formation and alter lung function.

Albert analyzed the effect of mechanical ventilation on surfactant function and considered the possibility that certain ventilation strategies cause the acute respiratory distress syndrome (18). He noted that experimental studies have demonstrated that mechanical ventilation for short periods of time increased lung elastic recoil, opening pressures, and surface tension of lung extracts and could cause atelectasis. These changes were directly related to the size of the tidal volume and inversely related to the PEEP level. He noted that some studies indicate the compression of the surfactant to less than $50 \%$ of its initial area resulted in film rupturing on reexpansion and increased surface tension. This problem is potentially more important in clinical syndromes associated with heterogeneous lung involvement with either inflammation or edema. These lungs would have alveoli with different sizes, different concentrations of surfactant, and different changes in alveolar pressure during inflation during mechanical ventilation.

In summary, the formation of alveolar edema secondary to heart failure, high oxygen concentrations in alveoli, and mechanical ventilation could alter surfactant function and change lung mechanics. These changes would affect gas exchange, cause hypoxemia, and could cause persistent dyspnea after diuresis. The non-uniformity of abnormal mechanics would also lead to ventilation/perfusion mismatching, further worsening hypoxemia.

\section{Surfactant in clinical studies}

Shimura et al. measured surfactant apoprotein A 
concentrations in airway secretions from 11 patients with cardiogenic pulmonary edema and 7 patients with chronic stable congestive heart failure (19). Surfactant apoprotein A levels were increased in patients with cardiogenic pulmonary edema with a mean concentration of $1,324 \mathrm{mcg}$ per $\mathrm{ml}$. The mean concentration in stable congestive heart failure patients was $78 \mathrm{mcg}$ per $\mathrm{mL}$. Surfactant protein A levels fell in all patients over six days except in three patients who died. There was also a significant positive relationship between the $\log$ of the surfactant protein A concentration and the mean capillary wedge pressure $(\mathrm{r}=0.78)$.

Günther et al. measured protein concentrations, differential cell counts, phospholipid concentrations, the percentage of lipid in large surfactant aggregates as an estimate of surfactant concentration, and surfactant activity in bronchoalveolar lavage fluid from patients with acute respiratory failure requiring mechanical ventilation (20). Patients with cardiogenic pulmonary edema had increased protein levels in their lavage fluid but no change in their lipid levels. These patients had normal surfactant activity in the large surfactant aggregates collected by centrifugation. However, when the protein in supernatants was added back to the individual large aggregate fractions, surface tension increased. This report demonstrates that patients with cardiogenic pulmonary edema have abnormal amounts of protein in alveoli secondary to barrier disruption; this could increase surface tension activity (an adverse effect) and increase fluid flux into alveoli.

\section{Surfactant release during episodes of pulmonary edema}

\section{Acute respiratory failure including cardiogenic pulmonary edema}

Surfactant protein concentrations are much higher in alveoli than in plasma. Consequently, changes in circulating plasma levels of surfactant proteins should reflect injury to blood gas barrier and transfer of surfactant into the systemic circulation. Doyle et al. measured surfactant protein A levels in plasma in patients with acute respiratory failure secondary to either acute cardiogenic pulmonary edema or adult respiratory distress syndrome (21). These levels were significantly higher than in control patients who required mechanical ventilation but did not have cardiorespiratory disease. There was a relationship between the surfactant protein A level and the degree of gas exchange impairment based on the $\mathrm{PaO}_{2} / \mathrm{FiO}_{2}$ ratio and lung mechanics based on compliance. Patients with reduced compliance and abnormal gas exchange had higher serum levels.

De Pasquale et al. measured surfactant protein A and surfactant protein B levels in 28 patients hospitalized with acute cardiogenic pulmonary edema (2). Both levels were increased and peaked on day 3 of hospitalization and remained above control levels on day 14 post presentation. Clinical improvement was obvious in most patients after two hours of treatment; radiographic improvement occurred over the next 3 days of hospitalization. Tumor necrosis factor alpha levels were also increased in these patients, peaked on day 1 , and stayed elevated over control values during the 14-day observation period. These authors concluded that the high capillary pressures associated with acute pulmonary edema caused stress failure of the pulmonary capillaries and barrier disruption in alveolar capillary units and that this created an acute inflammatory process that caused sustained injury over the 2 weeks following presentation.

Pappas and Filippatos summarized studies on pulmonary edema and acute heart failure in an editorial (1). They noted that the analysis of fluid flux based on the Starling equation did not completely explain the development of pulmonary edema in patients with acute heart failure and that there may be a disconnect between the level of dyspnea and pulmonary capillary wedge pressures. Increased capillary pressures can cause stress failure in barrier function in the lung that could stimulate both inflammatory and oxidative lung injury. This creates more barrier damage and increases permeability. Consequently, fluid moving out of the capillary into the interstitium and alveolar spaces reflects both hydrostatic gradients and changes in lung permeability. In addition, lymphatics and epithelial ion channels transfer fluid from the interstitium and alveoli back into the central venous circulation, and alterations in these tissues could also influence the development and recovery from pulmonary edema. Consequently, high surfactant protein levels in plasma reflect alveolar capillary barrier injury and provide an index of the duration of injury; measurement of surfactant levels could be incorporated into clinical management strategy in patients with cardiogenic pulmonary edema. 


\section{Chronic beart failure}

De Pasquale et al. measured plasma surfactant protein B levels in 53 patients with chronic heart failure and 19 control patients (22). Surfactant protein B levels were increased in patients with and correlated with the clinical severity of the heart failure. In addition, surfactant protein B levels increased in patients who deteriorated as outpatients and required an increase in diuretic dose, and levels decreased in patients who improved as outpatients and required a decrease in diuretic dose. Surfactant B protein levels also predicted hospitalization. These investigators suggested that surfactant protein B levels provides information about the effect of chronically elevated capillary pressures on alveolar capillary integrity. De Pasquale et al. also measured atrial natriuretic peptide and surfactant protein $B$ before and after treadmill exercise with stress echocardiography (23). Patients with positive stress echocardiograms during treadmill exercise with a mean heart rate of 99 beats per minute at the end of exercise had a significant increase in surfactant protein B. Consequently, they determined that patients with exerciseinduced myocardial dysfunction have detectable increases in surfactant protein B and presumably minor changes in alveolar capillary barriers associated with increased microvascular pressure during short periods of exercise.

Magrì et al. measured surfactant protein $\mathrm{B}$ levels in 71 patients with chronic heart failure and 19 healthy control subjects who underwent pulmonary function testing and maximum cardiopulmonary exercise tests (24). Surfactant protein B levels were increased in patients with heart failure, and increased surfactant protein B levels were associated with decreased membrane diffusion capacity on pulmonary function testing, decreased peak $\mathrm{O}_{2}$ consumption on exercise testing, and an increased slope of ventilation to $\mathrm{CO}_{2}$ production $\left(\mathrm{VE} / \mathrm{VCO}_{2}\right)$ during exercise. They concluded that higher circulating surfactant protein B levels were associated with damage to membranes involved in alveolar gas diffusion.

Gargiulo et al. measured "immature" surfactant protein $B$, mature surfactant protein A, mature surfactant protein $\mathrm{B}$, mature surfactant protein $\mathrm{D}$, and receptor for advanced glycation end products (RAGE) in 89 patients with chronic heart failure (25). Inclusion criteria included reduced left ventricular systolic function (ejection fraction less than $45 \%$ ), optimal medical management, and a stable clinical condition for at least 2 months. Patients had increased levels of immature surfactant protein $B$, surfactant protein $\mathrm{A}$, and surfactant protein $\mathrm{D}$ but did not have increased levels of mature surfactant protein B or RAGE. They had significant reductions in forced vital capacity, FEV1, diffusion capacity, peak workload on exercise testing, and peak $\mathrm{O}_{2}$ consumption. Elevated levels of immature surfactant protein $\mathrm{B}$ were strongly associated with a reduced diffusion capacity. They concluded that immature surfactant protein B is biological marker for of alveolar capillary membrane damage patients with chronic heart failure. They also reported that immature surfactant protein B predicted future hospitalization for heart failure (26). In summary, these four studies demonstrate that the measurement of surfactant levels when patients with chronic heart disease have potential clinical relevance in their management.

\section{Human pathology}

Lee studied lung biopsies using electron microscopy from 13 patients with chronic pulmonary edema secondary to cardiac disease (27). He found increased numbers of type II alveolar cells and irregular thickening of alveolar epithelial and capillary basement membranes. Fragmentation of the capillary basement membrane was seen only in patients with mean pulmonary arterial wedge pressures of greater than $35 \mathrm{mmHg}$ and heart failure for longer than 6 years. He described detachment of alveolar epithelial cells from the basement membrane, but widespread disruptions disruption of the alveolar epithelium did not occur. This study suggested adaptation or remodeling occur. More type II alveolar cells could increase surfactant production which helps stabilize alveolar spaces and could increase the transport of alveolar fluid out of the alveoli through the various ion channels in these cells. Haworth et al. collected lung biopsies from 15 adolescents who had rheumatic mitral stenosis undergoing cardiac surgery (28). Vascular abnormalities were most marked in the capillary bed and in the vessels immediately proximal and distal to the capillary. Capillaries had a marked increase in basement membranes and were embedded in a dense connective tissue within the alveolar wall. The peripheral airways were also encased in dense connective tissue which appeared to compress them, and bronchial smooth muscle was increased.

These two studies indicate that patients with chronically elevated left atrial and pulmonary capillary pressures have remodeling in alveolar capillary units with increased amounts of basement membrane and increased numbers of alveolar type II cells. These changes should help protect these units from disruption from elevated capillary pressures, increase surfactant levels to help maintain 
alveolar stability, and increase the number of ion channels necessary to transfer fluid out of alveolar spaces.

\section{Integrated overview of edema formation and clinical implications}

Increased hydrostatic pressures in pulmonary capillaries increase fluid flux across the endothelium into the interstitial spaces. At low rates of fluid formation, this fluid moves into the axial bronchial-vascular bundles and into pulmonary lymphatics. However, at higher rates of fluid formation, fluid enters the alveolar space. This fluid is transferred out of alveoli through sodium channels and aquaporins back into the interstitium. Edema fluid in the alveolar space alters surfactant function and increases surface tension. This increases hydrostatic gradients for fluid movement out of the capillaries into the interstitium and alveoli. In addition, it increases the likelihood of alveolar collapse at low lung volumes and creates abnormal gas exchange. High hydrostatic capillary pressures can cause barrier disruption which increases permeability in the alveolar epithelium and the capillary endothelium. This promotes fluid flux out of the capillaries at all hydrostatic capillary pressures. In addition, protein movement out of the capillaries into the interstitium and alveolar spaces increases surface tension. Barrier disruption creates an acute inflammatory process which may also injure lung tissue and sustain increases in permeability and cause direct surfactant dysfunction.

Consequently, acute increases in capillary pressure can cause lung injury and barrier disruption and promote fluid formation both through hydrostatic mechanisms and changes in permeability. Patients with both acute cardiogenic pulmonary edema and with chronic heart failure have high plasma levels of surfactant protein B which represents barrier disruption. Positive pressure ventilation can also cause lung injury and surfactant dysfunction, and high concentrations of oxygen in alveolar spaces could potentially alter surfactant function. Patients with chronic heart failure have a thickened barrier in the alveolar capillary wall, increased numbers of alveolar type II cells, and increased surfactant in alveoli. These adaptive responses help protection against fluid accumulation but also delay clearance when fluid does accumulate. Consequently, the management of patients with acute and chronic pulmonary edema needs attention to both capillary pressures and lung injury mechanisms. Abnormal chest X-rays may reflect both pulmonary edema and lung injury; diuresis may not clear pulmonary infiltrates in some patients.

\section{Acknowledgments}

None.

\section{Footnote}

Conflicts of Interest: The authors have no conflicts of interest to declare.

Ethical Statement: The authors are accountable for all aspects of the work in ensuring that questions related to the accuracy or integrity of any part of the work are appropriately investigated and resolved.

\section{References}

1. Pappas L, Filippatos G. [Pulmonary congestion in acute heart failure: from hemodynamics to lung injury and barrier dysfunction]. Rev Esp Cardiol 2011;64:735-8.

2. De Pasquale CG, Arnolda LF, Doyle IR, et al. Prolonged alveolocapillary barrier damage after acute cardiogenic pulmonary edema. Crit Care Med 2003;31:1060-7.

3. Weibel ER. What makes a good lung? Swiss Med Wkly 2009;139:375-86.

4. West JB. Invited review: pulmonary capillary stress failure. J Appl Physiol (1985) 2000;89:2483-9; discussion 2497.

5. Bachofen H, Schürch S, Michel RP, et al. Experimental hydrostatic pulmonary edema in rabbit lungs. Morphology. Am Rev Respir Dis 1993;147:989-96.

6. Bachofen H, Schürch S, Weibel ER. Experimental hydrostatic pulmonary edema in rabbit lungs. Barrier lesions. Am Rev Respir Dis 1993;147:997-1004.

7. Tsukimoto K, Mathieu-Costello O, Prediletto R, et al. Ultrastructural appearances of pulmonary capillaries at high transmural pressures. J Appl Physiol (1985) 1991;71:573-82.

8. Fu Z, Costello ML, Tsukimoto K, et al. High lung volume increases stress failure in pulmonary capillaries. J Appl Physiol (1985) 1992;73:123-33.

9. Elliott AR, Fu Z, Tsukimoto K, et al. Short-term reversibility of ultrastructural changes in pulmonary capillaries caused by stress failure. J Appl Physiol (1985) 1992;73:1150-8.

10. West JB, Mathieu-Costello O, Jones JH, et al. Stress failure of pulmonary capillaries in racehorses with exerciseinduced pulmonary hemorrhage. J Appl Physiol (1985) 1993;75:1097-109.

11. Hopkins SR, Schoene RB, Henderson WR, et al. Intense 
exercise impairs the integrity of the pulmonary bloodgas barrier in elite athletes. Am J Respir Crit Care Med 1997;155:1090-4.

12. Said SI, Avery ME, Davis RK, et al. Pulmonary surface activity in induced pulmonary edema. J Clin Invest 1965;44:458-64.

13. Albert RK, Lakshminarayan S, Hildebrandt J, et al. Increased surface tension favors pulmonary edema formation in anesthetized dogs' lungs. J Clin Invest 1979;63:1015-8.

14. Bredenberg CE, Nieman GF, Paskanik AM, et al. Microvascular membrane permeability in high surface tension pulmonary edema. J Appl Physiol (1985) 1986;60:253-9.

15. Nieman GF, Bredenberg CE. High surface tension pulmonary edema induced by detergent aerosol. J Appl Physiol (1985) 1985;58:129-36.

16. Nieman GF, Bredenberg CE, Paskanik AM. Positive endexpiratory pressure accelerates lung water accumulation in high surface tension edema. Surgery 1990;107:156-62.

17. Smallwood CD, Boloori-Zadeh P, Silva MR, et al. High Oxygen Concentrations Adversely Affect the Performance of Pulmonary Surfactant. Respir Care 2017;62:1085-90.

18. Albert RK. The role of ventilation-induced surfactant dysfunction and atelectasis in causing acute respiratory distress syndrome. Am J Respir Crit Care Med 2012;185:702-8.

19. Shimura S, Masuda T, Takishima T, et al. Surfactant apoprotein-A concentration in airway secretions for the detection of pulmonary oedema. Eur Respir J 1996;9:2525-30.

20. Günther A, Siebert C, Schmidt R, et al. Surfactant alterations in severe pneumonia, acute respiratory distress

Cite this article as: Nugent K, Dobbe L, Rahman R, Elmassry M, Paz P. Lung morphology and surfactant function in cardiogenic pulmonary edema: a narrative review. J Thorac Dis 2019;11(9):4031-4038. doi: 10.21037/jtd.2019.09.02 syndrome, and cardiogenic lung edema. Am J Respir Crit Care Med 1996;153:176-84.

21. Doyle IR, Nicholas TE, Bersten AD. Serum surfactant protein-A levels in patients with acute cardiogenic pulmonary edema and adult respiratory distress syndrome. Am J Respir Crit Care Med 1995;152:307-17.

22. De Pasquale CG, Arnolda LF, Doyle IR, et al. Plasma surfactant protein-B: a novel biomarker in chronic heart failure. Circulation 2004;110:1091-6.

23. De Pasquale CG, Arnolda LF, Doyle IR, et al. Circulating surfactant protein-B levels increase acutely in response to exercise-induced left ventricular dysfunction. Clin Exp Pharmacol Physiol 2005;32:622-7.

24. Magrì $\mathrm{D}$, Brioschi $\mathrm{M}$, Banfi $\mathrm{C}$, et al. Circulating plasma surfactant protein type B as biological marker of alveolarcapillary barrier damage in chronic heart failure. Circ Heart Fail 2009;2:175-80.

25. Gargiulo P, Banfi C, Ghilardi S, et al. Surfactant-derived proteins as markers of alveolar membrane damage in heart failure. PLoS One 2014;9:e115030.

26. Magrì D, Banfi C, Maruotti $\mathrm{A}$, et al. Plasma immature form of surfactant protein type B correlates with prognosis in patients with chronic heart failure. A pilot single-center prospective study. Int J Cardiol 2015;201:394-9.

27. Lee YS. Electron microscopic studies on the alveolarcapillary barrier in the patients of chronic pulmonary edema. Jpn Circ J 1979;43:945-54.

28. Haworth SG, Hall SM, Panja M, et al. Peripheral pulmonary vascular and airway abnormalities in adolescents with rheumatic mitral stenosis. Int J Cardiol 1988;18:405-16. 